\title{
SHUT-OFF VALVES OF ELECTRIC DRIVE WITH THYRISTOR VOLTAGE REGULATORS AND SOFTWARE IMPLEMENTATION OF THE INDIRECT DETERMINATION OF MOMENT
}

\author{
Anton I. Vlasov ${ }^{1}$, Vladislav. Yu. Dementyev ${ }^{2}$, Alexandr. S. Karakylov ${ }^{1, *}$, and \\ Leonid. S. Udut ${ }^{1}$ \\ ${ }^{1}$ Tomsk Polytechnic University, 634050, Tomsk, Russia \\ ${ }^{2}$ Specialist of 1 st class of production unit of "Gazprom Transgaz Tomsk", Tomsk, Russia
}

\begin{abstract}
In this paper we consider the shut-off valve of electric drive with thyristor voltage regulator and microprocessor control which ensures a constant indirect control of torque value on the electric drive as well as its limitation in the case of the creation of an emergency on the main oil pipeline. It was found that the use of the integrated microprocessor control systems with software implementation calculating of given torque in the system of induction motor - thyristor voltage regulator, allows you to get the best performance of electric drive shut-off valve with the restrictions on the maximum current, torque whith deviations of voltage parameters and improve the safety of the process of oil pumping.
\end{abstract}

\section{Introduction}

The development of the fuel and energy complex of our country is largely dependents on the operation of pipeline transport, in particular the main oil pipelines. Main oil pipeline is the cheapest means of delivery of oil and oil products from their place of production to refineries. For effective work of the oil pipeline and constant control of the of the oil transportation used shut-off valves, the main purpose of which - to block fluid flow in a pipe by locking valve body, and again let the medium depending on the requirements of the process, according to an accepted conduit. The drive of shut-off valves was designed to move the locking bolt body in end positions, as well as the establishment, where necessary, efforts to achieve the desired tightness of the seal.

At the moment, as the motorized shut-off valves apply electric drive with a frequency converter and a thyristor voltage regulator. The main advantages of the inverter are the high control accuracy, large range. Application of the frequency converter is not limited, so at the modernization and introduction of new technologies they are used everywhere [1]. However, the mechanisms that do not require a large adjustment range, but need a soft start and accurate stop, possible to use a thyristor voltage regulators of trimmer. The main advantage of thyristor voltage compared with a frequency converter is its low cost and high reliability $[2,4]$.

\footnotetext{
${ }^{*}$ Corresponding author: karakulov@,tpu.ru
} 
Ensuring the safety of technological process of oil transporting is only possible with the high reliability of the drive The failures occur primarily in the power control unit coz of external factors (low supply voltage, imbalance voltage of one phase, and etc.). Therefore, the creation of the electric shut-off valves to operate at a reduced supply voltage, as well as asymmetrical modes of electric grid operation, is today an urgent problem [3, 4].

The purpose of this article is to develop electric drive with thyristor voltage regulator and microprocessor control provides constant control the value of output torque of the drive as well as its limitation in the case of the creation of an emergency on the main oil pipeline, for example, the electric shut-off valve at lower supply voltage, as well as the unbalance in one phase of the mains supply.

\section{Study technique}

To carry out such a task often used computational methods based primarily on modeling modes of operation of the induction motor (IM). However, they are computing algorithm repeated with high frequency in real time and require significant resources of the microprocessor system. In addition, the accuracy of these methods depends on the accuracy of determining IM parameters. The use of an alternative method for the indirect determination of output torque IM [4, 5], implemented on the basis of microprocessor control unit with thyristor voltage regulator, allows the use of less powerful microprocessors, making the system electric shut-off valve cheaper, and therefore more competitive on the drive technology market shut-off valves. As is well known [6, 7], in static mods, torque on the shaft of induction motor can be represented as a function of the current values of the stator current (with electric drive movement) or as a function of the steering angle of the thyristors voltage. And for each of the stator voltages, the data will look different curves, so we are dealing with the group characteristics. Therefore, if in three dimensions on the axes postpone current, voltage and torque we get a surface that describes the time induction motor as a function of two variables: current and voltage (Fig. 1, A). Consequently, the same three-dimensional surface we can get if we represent time as a function of voltage and controls the angle of the thyristors by thyristor voltage regulator (Fig. $1 \mathrm{~b}$ )
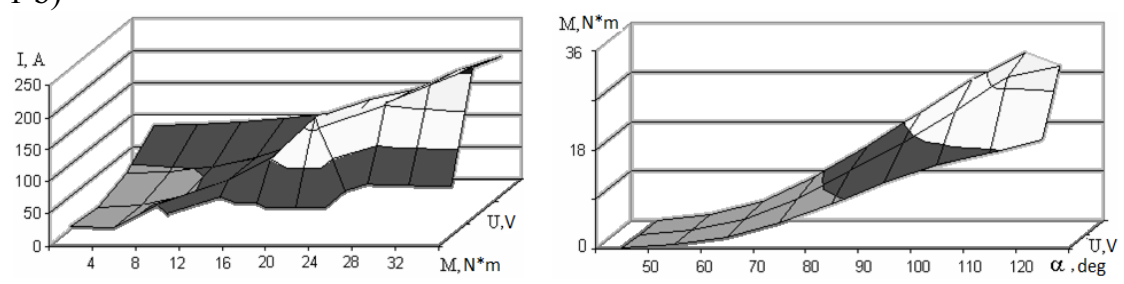

Fig. 1. Dependence of the torque developed by induction motor:a) the stator current and suply voltage b) opening angle of thyristors and the supply voltage.

The asymmetry (imbalance) electric phase grid causes fluctuations of currents and voltages, which affects on the output signal extracted from the current and voltage sensors, which will be complex. In this case it is necessary to interpolate information from current and voltage sensors and with the help of three-dimensional surfaces to determine torque setpoint, providing the maximum possible speed of the shut-off valve body.

Fig. 2 shows a graphical interpretation of the algorithm for torque calculating, which used in the microprocessor control unit - thyristor voltage regulator. Here: the characteristic described by the equation $\mathrm{M}_{2}=\mathrm{kI}_{2}+\mathrm{b}$ matches to the values of the torque at nominal supply voltage, by the equation $\mathrm{M}_{1}=\mathrm{kI}_{1}+\mathrm{b}$ - the values of the torque when an imbalance in 
one of the phases of the grid and the equation $\mathrm{M}=\mathrm{kI}+\mathrm{b}$ - the values at the torque of any supply condition.

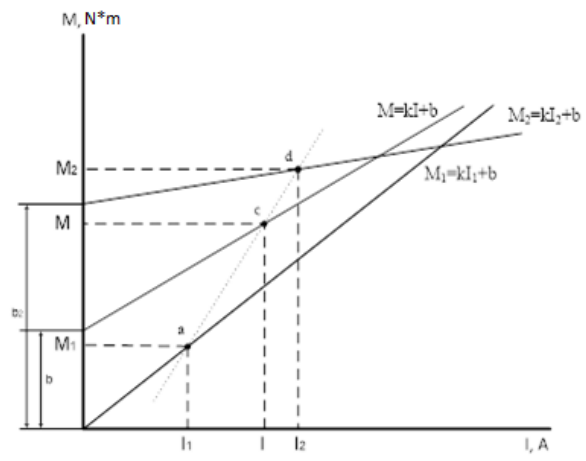

Fig. 2. Graphical interpretation of calculation of the set torque by the microprocessor control unit.

If, at a certain time, it is necessary to have information about the current value (point "c" in Fig. 2) matching to a given torque on the linear characteristic $\mathrm{M}=\mathrm{kI}+\mathrm{b}$ through the predetermined point of a straight line intersecting characteristics $\mathrm{M}_{2}=\mathrm{kI}_{2}+\mathrm{b}$ and $\mathrm{M}_{1}=\mathrm{kI}_{1}+\mathrm{b}$ at points "a" and " $\mathrm{d}$ ", which are known. Using the method of interpolating rectangular, one can determine the coordinate point "a" (in this case, the required current value I) according to following equation

$$
\mathrm{I}=\mathrm{M}_{1}+\frac{\mathrm{M}_{2}-\mathrm{M}_{1}}{\mathrm{I}_{2}-\mathrm{I}_{1}} \cdot\left(\mathrm{M}-\mathrm{M}_{1}\right)
$$

The parameter $b$ characteristics of the torque is the ratio of the imbalance voltage of the nominal voltage and matches necessary coordinate of torque value on the $y$-axis of the torque characteristic.

\section{Conclusion}

The use of the integrated microprocessor control systems with software implementation calculating with a given torque in the system of induction motor - thyristor voltage regulator allows you to get the best performance shut-off valve electric drive with with the restrictions on the Overcurrent, the torque when deviations of parameters voltage and improve the safety of the process of oil pumping.

\section{References}

1. Y.N. Dementyev, K.N. Negodin, N.V. Koyain, L.S. Udut, Proceedings of the Higher Educational Institutions. Electromechanics, 4, 49 (2016)

2. V.A. Shybenko, N.Y. Braslavsky, Thyristor asynchronous electric drive with phase control (Energy, St. Petersburg, 1972) [in Russian]

3. A.S. Karakulov, the fourth Scientific Conference Modern Equipment and the Automation System, 63 (2004)

4. A.S. Karakulov, V.V. Krasilyev, S.V. Langraf, A.Y. Marjanov, the V Interdisciplinary Scientific-Technical Conference Automation and Advanced Technologies, (2005)

5. Y.N. Dementyev, A.D. Umurzakova, MATEC Web of Conf.,19, 01027 (2014)

6. Y.N. Dementiev, A.Y. Chernyshev, I.A. Chernyshev, Electric drive (Yurayt, Moscow, 2016) [in Russian]

7. R.F. Bekishev, Y.N. Dementiev, Power (Yurayt, Moscow, 2016) [in Russian] 